\section{Reply to Aron R Kater et al.}

We do wish to thank Kater et al. for their interest in our article about BIRC3 mutations in fludarabine, cyclophosphamide and rituximab (FCR) treated chronic lymphocytic leukemia (CLL) patients and for expanding the knowledge on the clinical implications of BIRC 3 mutations in the context of novel biological drugs.

We conducted a retrospective multicenter real life study on 287 CLL patients treated with first line FCR and observed that BIRC3 mutated patients experienced a poor outcome, superimposable to that of patients with TP53 disruption, that represents the strongest marker of chemorefractoriness. ${ }^{2}$ Our initial results, corroborated also by other prospective trials and complemented with in vitro evidence, validated BIRC3 as a prognostic biomarker after chemoimmunotherapy in CLL. ${ }^{1-}$

A prognostic biomarker is a biological feature of the tumor that provides information about the disease natural history independent of the treatment received. However, in order to gain solid clinical relevance, a biomarker should also be provided with a predictive value that informs about the likely benefit from a specific treatment. ${ }^{5,6}$ Currently, TP53 abnormalities and immunoglobulin variable heavy chain gene (IGHV) mutational status fulfill the criteria of predictive biomarkers whose usage is recommended by guidelines for the clinical management and treatment choice of CLL patients.

Molecular studies of phase 3 randomized clinical trials are essential to transform a prognostic biomarker into a predictive biomarker by showing the interaction between the biomarker and treatment. In that sense, BIRC3 mutations are a validated prognostic biomarker since they associate with shorter progression free survival when patients are treated with chemoimmunotherapy, but not when treated with fixed duration venetoclax in combination with anti-CD20 monoclonal antibody. In this issue of the journal, Kater et al. report the initial results of the molecular analysis of the MURANO trial dedicated to relapsed/refractory CLL, and show that BIRC3 mutated patients treated with bendamustine rituximab experienced a worse outcome compared to wildtype patients. Conversely, the combination of venetoclax with rituximab was able to overcome the negative impact of BIRC3 mutations (Table 1). ${ }^{1}$ Similarly, the companion biomarker study of the CLL14 trial indicated that, also in the first line setting, obinutuzumab-venetoclax, but not obinutuzumab-chlorambucil is an effective therapeutic option for BIRC3 mutated patients (Table 1).

Whereas the efficacy of venetoclax in overcoming $B I R C 3$ disruption is validated in two trials, the role of ibrutinib in this context is still unexplored. Mantle cell lymphomas carrying BIRC3 mutations appear to be resistant to ibrutinib in vitro, but in vivo studies are needed to confirm this pre-clinical information. ${ }^{8}$ Although several phase 3 clinical trials have demonstrated the superiority of ibrutinib versus chemoimmunotherapy in CLL, the predictive value of $B I R C 3$ mutations has not been tested to date in this context. ${ }^{9-11}$

The introduction of Bruton tyrosine kinase inhibitors and of venetoclax have changed the natural history of CLL. Despite these advantages, molecular predictors, namely IGHV mutational status and TP53 disruption, are still essential in treatment choices. In this context, BIRC 3 is emerging as a novel predictive biomarker that might enter the routine clinical practice allowing a better treatment algorithm for every individual CLL patient.

\section{Riccardo Moia, ${ }^{1}$ Gianluca Gaidano ${ }^{1}$ and Davide Rossi ${ }^{2}$}

${ }^{2}$ Division of Hematology, Department of Translational Medicine, Università del Piemonte Orientale, Novara, Italy and Institute of Oncology Research and Oncology Institute of Southern Switzerland, Bellinzona, Switzerland

Correspondence:DAVIDE ROSSI - davide.rossi@eoc.ch

doi:10.3324/haematol.2020.255596

Information on authorship, contributions, and financial \& other disclosures was provided by the authors and is available with the online version of this article at wWw. haematologica.org.

\section{References}

1. Kater AP, Jiang Y, Chyla B, et al. Response in patients with BIRC3mutated relapsed/refractory chronic lymphocytic leukemia treated with fixed-duration venetoclax and rituximab. Haematologica. 2020;105(7):382-383.

2. Diop F, Moia R, Favini C, et al. Biological and clinical implications of BIRC3 mutations in chronic lymphocytic leukemia. Haematologica. 2020;105(2):448-456.

3. Estenfelder S, Tausch E, Robrecht S, et al. Gene mutations and treatment outcome in the context of chlorambucil (Clb) without or with the addition of rituximab (R) or obinutuzumab (GA-101, G) - results of an extensive analysis of the Phase III Study CLL11 of the German CLL Study Group. Blood. 2016;128(22):3227.

4. Tausch E, Schneider C, Robrecht S, et al. Prognostic and predictive impact of genetic markers in patients with CLL treated with obinutuzumab and venetoclax. Blood. 2020 Mar 23. [Epub ahead of print].

5. Rossi D, Gerber B, Stüssi G. Predictive and prognostic biomarkers in the era of new targeted therapies for chronic lymphocytic leukemia. Leuk Lymphoma. 2017;58(7):1548-1560.

6. Ballman KV. Biomarker: predictive or prognostic? J Clin Oncol. 2015; 33(33):3968-3971

7. Hallek M, Cheson BD, Catovsky D, et al. iwCLL guidelines for diagnosis, indications for treatment, response assessment, and supportive management of CLL. Blood. 2018;131(25):2745-2760.

8. Rahal R, Frick M, Romero R, et al. Pharmacological and genomic profiling identifies NF- $\kappa \mathrm{B}$-targeted treatment strategies for mantle cell lymphoma. Nat Med. 2014;20(1):87-92.

9. Shanafelt TD, Wang XV, Kay NE, et al. Ibrutinib-rituximab or chemoimmunotherapy for chronic lymphocytic leukemia. N Engl J Med. 2019;381(5):432-443.

10. Woyach JA, Ruppert AS, Heerema NA, et al. Ibrutinib regimens versus chemoimmunotherapy in older patients with untreated CLL. N Engl J Med. 2018;379(26):2517-2528.

11. Moreno C, Greil R, Demirkan F, et al. Ibrutinib plus obinutuzumab versus chlorambucil plus obinutuzumab in first-line treatment of chronic lymphocytic leukaemia (iLLUMINATE): a multicentre, randomised, open-label, phase 3 trial. Lancet Oncol. 2019;20(1):43-56.

Table 1. Clinical impact of BIRC3 mutations in the MURANO and in the CLL14 trial.

\begin{tabular}{|c|c|c|c|c|}
\hline IIrial & Phase & Setting & Interventions & BIRC3 mutations \\
\hline \multirow[t]{2}{*}{ MURANO trial ${ }^{1}$} & 3 & Relapsed/refractory CLL patients & Venetoclax + Rituximab & HR 1.50 (95\% CI: $0.50-4.30) P=0.44$ \\
\hline & & & Bendamustine + Rituximab & HR 2.20 (95\% CI: $0.92-5.10) P=0.077$ \\
\hline \multirow[t]{2}{*}{ CLL14 trial $^{4}$} & 3 & Untreated CLL patients & Venetoclax + Obinutuzumab & HR 1.10 (95\% CI: $0.15-8.13) P=0.92$ \\
\hline & & & Chlorambucil + Obinutuzumab & HR 4.03 (95\% CI: 1.73-9.37) $P<0.01$ \\
\hline
\end{tabular}

CLL: chronic lymphocytic leukemia; HR: hazard ratio; CI: confidence interval; $P$ : $P$-value. 